Available online at http://jnfa.mathres.org
MATHES Journal of Nonlinear Functional Analysis
Ants

\title{
HIGHER ORDER FRACTIONAL BOUNDARY VALUE PROBLEMS FOR MIXED TYPE DERIVATIVES
}

\author{
RABAH KHALDI, ASSIA GUEZANE-LAKOUD* \\ Laboratory of Advanced Materials, Department of Mathematics, Faculty of Sciences, \\ Badji Mokhtar Annaba University, P.O. Box 12, Annaba, 23000, Algeria
}

\begin{abstract}
Some results on the existence of solutions for a nonlinear higher order fractional differential equation involving both the left Riemann-Liouville and the right Caputo fractional derivatives with a natural boundary condition are obtained. The results presented in this paper are based on the method of upper and lower solutions and the monotonicity of the right Caputo derivative. Moreover, we give the explicit expression of the lower and upper solutions. Two illustrative numerical examples are also provided.
\end{abstract}

Keywords. Boundary value problem; Fractional derivative; Method of upper and lower solutions; Existence of solutions.

2010 Mathematics Subject Classification. 34A08, 34B15.

\section{Introduction}

Recently, the study of nonlinear fractional differential equations has attracted much attention of researchers and different methods have been investigated; [1]-[11] and the references therein. However, few works exist in the literature concerning nonlinear fractional boundary value problems with mixed fractional derivatives. In [4], Blaszczyk studied numerically a linear fractional oscillator equation involving both the right Caputo and the left Riemann-Liouville derivatives of order $\alpha \in(0,1)$ subject to a natural condition. In [6], Guezane-Lakoud, Khaldi and Torres

\footnotetext{
${ }^{*}$ Corresponding author.
}

E-mail addresses: rkhadi@yahoo.fr (R. Khaldi), a_guezane@yahoo.fr (A. Guezane-Lakoud).

Received January 19, 2017; Accepted May 10, 2017. 
proved the existence of solutions for a nonlinear fractional oscillator equation with both left Riemann-Liouville and right Caputo fractional derivatives subject to natural boundary conditions via the method of lower and upper solutions. In [2], Agrawal investigated some fractional variational problems defined in terms of both the Riemann-Liouville and the Caputo derivatives and presented the generalized Euler-Lagrange equations and the transversality conditions.

In this paper, we focus on the following sequential higher order fractional boundary value problem (P1) involving both the Riemann-Liouville and the Caputo types fractional derivatives:

$$
(-1)^{m C} D_{1^{-}}^{\alpha} D_{0^{+}}^{\beta} u(t)+f(t, u(t))=0,0 \leq t \leq 1,
$$

with initial conditions

$$
u(0)=u^{(i)}(0)=0, i=1, \ldots, m+n-2,
$$

and natural condition (see [4])

$$
D_{0^{+}}^{\beta+m-1} u(1)=0
$$

where $m-1<\alpha<m, n-1<\beta<n$, the integers $m$ and $n$ are such $m, n \geq 2,{ }^{C} D_{1^{-}}^{\alpha}$ denotes the right Caputo derivative, $D_{0^{+}}^{\beta}$ denotes the left Riemann-Liouville derivative, $u$ is the unknown function and $f \in C([0,1] \times \mathbb{R}, \mathbb{R})$.

By using the method of lower and upper solutions and the Schauder fixed point theorem, we prove the existence of solutions of problem (P1). The presence of both the right Caputo and the left Riemann-Liouville derivatives in the differential equation (1.1) leads to great difficulties in the proof of the existence of solutions. To overcome the difficulties, we transform the problem (P1) to an equivalent Caputo fractional boundary value problem of order $p, 0<p<1, p$ will be specified later. Then we construct the lower and upper solutions of problem (P1). Moreover, we use a new result on the monotonicity of the right Caputo derivative.

The method of upper and lower solutions has been applied in the investigation of the existence of solutions for nonlinear boundary value problems in many works; see $[6,7,12,13]$ and the references therein.

Next, we recall some essential definitions on fractional calculus $[8,10,11]$. 
Definition 1.1. Let $g$ be a real function defined on $[0,1]$ and $\mu>0$. Then the left and right Riemann-Liouville fractional integrals of order $\mu$ of $g$ are defined respectively by

$$
\begin{aligned}
I_{0^{+}}^{\mu} g(t) & =\frac{1}{\Gamma(\mu)} \int_{0}^{t} \frac{g(s)}{(t-s)^{1-\mu}} d s, \\
I_{1^{-}}^{\mu} g(t) & =\frac{1}{\Gamma(\mu)} \int_{t}^{1} \frac{g(s)}{(s-t)^{1-\mu}} d s .
\end{aligned}
$$

The left Riemann-Liouville fractional derivative and the right Caputo fractional derivative of order $\mu>0$ of function $g$ are respectively:

$$
\begin{aligned}
D_{0^{+}}^{\mu} g(t) & =\frac{d^{k}}{d t^{k}}\left(I_{0^{+}}^{k-\mu} g\right)(t), \\
{ }^{C} D_{1^{-}}^{\mu} g(t) & =(-1)^{k} I_{1^{-}}^{k-\mu} g^{(k)}(t),
\end{aligned}
$$

where $k$ is the smallest integer greater or equal than $\mu$.

The highlights of this article are as follows:

- We reduce mixed higher order boundary value problem (P1) to a lower order Caputo boundary value problem.

- We construct the lower and upper solutions of problem (P1).

- To prove the existence and localization of solutions of problem (P1), we construct a sequence of modified problems. Then we use a result on the monotonicity of the right Caputo derivative.

\section{Main results}

To prove the existence of solutions of problem (P1), we need the following result on the right Caputo derivative of monotone functions.

Theorem 2.1. [6] Assume that $0<s<1$ and $g \in C^{1}[0,1]$ is such that ${ }^{C} D_{1^{-}}^{s} g(t) \geq 0$ for all $t \in[0,1]$ and all $s \in(r, 1)$ with some $r \in(0,1)$. Then, $g$ is monotone decreasing. Similarly, if ${ }^{C} D_{1^{-}}^{s} g(t) \leq 0$ for all $t$ and $\mu$ mentioned above, then $g$ is monotone increasing.

Define the space

$$
\begin{aligned}
A C^{n+m}[0,1]= & \left\{u \in C^{n+m-1}[0,1]\right. \\
& \left.u^{(n+m-1)} \text { absolutely continuous function on }[0,1]\right\} .
\end{aligned}
$$


Now, taking into account Theorem 2.1, we update the definition of lower and upper solutions of problem (P1) as follows:

Definition 2.2. The functions $\underline{\sigma}, \bar{\sigma} \in A C^{n+m}[0,1]$ are called lower and upper solutions of problem $(\mathrm{P} 1)$ respectively, if

a) $(-1)^{m C} D_{1^{-}}^{\alpha_{0}} D_{0^{+}}^{\beta} \underline{\sigma}(t)+f(t, \underline{\sigma}(t)) \leq 0$, for all $t \in[0,1]$ and $\alpha_{0} \in[\alpha, m)$,

$\underline{\sigma}(0) \geq 0, \underline{\sigma}^{(i)}(0) \geq 0, i=1, \ldots, m+n-2, D_{0^{+}}^{\beta+m-1} \underline{\sigma}(1) \geq 0$.

b) $(-1)^{m C} D_{1^{-}}^{\alpha_{0}} D_{0^{+}}^{\beta} \bar{\sigma}(t)+f(t, \bar{\sigma}(t)) \geq 0$, for all $t \in[0,1]$ and $\alpha_{0} \in[\alpha, m)$,

$\bar{\sigma}(0) \leq 0, \bar{\sigma}^{(i)}(0) \leq 0, i=1, \ldots, m+n-2, D_{0^{+}}^{\beta+m-1} \bar{\sigma}(1) \leq 0$.

Functions $\underline{\sigma}$ and $\bar{\sigma}$ are called lower and upper solutions in reverse order if $\underline{\sigma}(t) \geq \bar{\sigma}(t)$, $0 \leq t \leq 1$

Remark 2.3. Denote $q=\alpha_{0}-m+1$ and $p=\alpha-m+1$. Then $0<q, p<1$. By using the properties of Caputo derivative, we prove that

$$
{ }^{C} D_{1^{-}}^{\alpha_{0}} u(t)=(-1)^{(m-1) C} D_{1^{-}}^{q} D^{m-1} u(t) .
$$

Consequently, conditions (a) and (b) in Definition 2.2 are equivalent to:

(a') ${ }^{C}{ }^{C} D_{1^{-}}^{q} D_{0^{+}}^{\beta+m-1} \underline{\sigma}(t)+f(t, \underline{\sigma}(t)) \leq 0$,for all $t \in[0,1]$ and $q \in[p, 1)$,

$\underline{\sigma}(0) \geq 0, \underline{\sigma}^{(i)}(0) \geq 0, i=1, \ldots, m+n-2, D_{0^{+}}^{\beta+m-1} \underline{\sigma}(1) \geq 0$.

(b') $-{ }^{C} D_{1^{-}}^{q} D_{0^{+}}^{\beta+m-1} \bar{\sigma}(t)+f(t, \bar{\sigma}(t)) \geq 0$,for all $t \in[0,1]$ and $q \in[p, 1)$.

$\bar{\sigma}(0) \leq 0, \bar{\sigma}^{(i)}(0) \leq 0, i=1, \ldots, m+n-2, D_{0^{+}}^{\beta+m-1} \bar{\sigma}(1) \leq 0$.

That means that if $\underline{\sigma}$ and $\bar{\sigma}$ are lower and upper solutions of problem (P1), they still should be lower and upper solutions for the sequence of boundary value problems generated by conditions (1.2)-(1.3) and the following fractional differential equations

$$
(-1)^{m C} D_{1^{-}}^{\alpha_{0}} D_{0^{+}}^{\beta} u(t)+f(t, u(t))=0,0 \leq t \leq 1,
$$

for all $\alpha_{0} \in[\alpha, m)$.

Next, we prove the existence of solutions of problem (P1). We assume that nonlinear term $f(t, x)$ lies between two curves for some $x$.

Theorem 2.4. Assume that there exist two constants $A \geq 0$ and $B \leq 0$ such that $|B| \leq A$ and the following hypotheses hold:

(H1) $f(t, x) \leq A(1-t)^{1-q}$, for $0 \leq t \leq 1,0 \leq x \leq \frac{A}{\Gamma(\beta+m)}$ and for all $q \in[p, 1)$.

(H2) $f(t, x) \geq B(1-t)^{1-q}$ for $0 \leq t \leq 1, \frac{B}{\Gamma(\beta+m)} \leq x \leq 0$ and for all $q \in[p, 1)$. 
Then problem (P1) has at least one solution u such that

$$
\begin{aligned}
\bar{\sigma}(t) & \leq u(t) \leq \underline{\sigma}(t) \\
D_{0^{+}}^{\beta+m-1} \bar{\sigma}(t) & \leq D_{0^{+}}^{\beta+m-1} u(t) \leq D_{0^{+}}^{\beta+m-1} \underline{\sigma}(t), 0 \leq t \leq 1
\end{aligned}
$$

where $\underline{\sigma}(t)$ and $\bar{\sigma}(t)$ are the lower and upper solutions respectively of problem $(P 1)$ with reversed order. Recall that $p=\alpha-m+1$ and $0<p<1$.

Proof. The proof is split in some steps. First, we begin by solving a Riemann-Liouville fractional boundary value problem that we denote by $(\mathrm{P} 2)$ :

$$
\begin{gathered}
D_{0^{+}}^{\beta+m-1} u(t)=v(t), 0 \leq t \leq 1, \\
u(0)=u^{(i)}(0)=0, i=1, \ldots, m+n-2 .
\end{gathered}
$$

Applying the properties of Riemann-Liouville fractional derivative and the initial conditions (2.2), we find that

$$
u(t)=\frac{1}{\Gamma(\beta+m-1)} \int_{0}^{t}(t-s)^{\beta+m-2} v(s) d s .
$$

Define the operator $T$ on $C([0,1], \mathbb{R})$ by

$$
T v(t)=\frac{1}{\Gamma(\beta+m-1)} \int_{0}^{t}(t-s)^{\beta+m-2} v(s) d s=I_{0^{+}}^{\beta+m-1} v(t), t \in[0,1] .
$$

Thus $u(t)=T v(t)$. By using the properties of Caputo derivative, we obtain that

$$
{ }^{C} D_{1^{-}}^{\alpha} u(t)=(-1)^{(m-1) C} D_{1^{-}}^{\alpha-m+1} D^{m-1} u(t)
$$

Taking into account natural condition (1.3), property (2.4) of Caputo derivative and RiemannLiouville derivative's property

$$
D^{m-1} D_{0^{+}}^{\beta} u(t)=D_{0^{+}}^{\beta+m-1} u(t)
$$

we conclude that problem (P1) is equivalent to the following Caputo boundary value problem of order $p=\alpha-m+1$, with $0<p<1$ :

$$
(P 3)\left\{\begin{array}{c}
-{ }^{C} D_{1^{-}}^{p} v(t)+f(t, T v(t))=0,0 \leq t \leq 1 \\
v(1)=0
\end{array}\right.
$$

Step 1. Existence of lower and upper solutions of problem (P1). 
Setting $\varphi(t)=A(1-t)$, we see that $\underline{\sigma}(t)=T \varphi(t)$ is a lower solution of problem (P1). In fact, we have

$$
\underline{\sigma}(t)=T \varphi(t)=I_{0^{+}}^{\beta+m-1} \varphi(t)=\frac{A t^{\beta+m-1}(\beta+m-t)}{\Gamma(\beta+m+1)} \leq \frac{A}{\Gamma(\beta+m)} .
$$

From Hypothesis (H1), we find, for all $q \in[p, 1)$,

$$
\begin{aligned}
& -{ }^{C} D_{1^{-}}^{q} \varphi(t)+f(t, T \varphi(t)) \\
= & \frac{-A}{\Gamma(2-q)}(1-t)^{1-q}+f(t, T \varphi(t)) \leq-A(1-t)^{1-q}+f(t, T \varphi(t)) \leq 0 .
\end{aligned}
$$

In addition, we also have $\underline{\sigma}(0)=T \varphi(0)=0, D_{0^{+}}^{\beta+m-1} \underline{\sigma}(1)=\varphi(1)=0$.

Similarly, set $\psi(t)=B(1-t)$. From Hypothesis $(\mathrm{H} 2)$, we obtain that $\bar{\sigma}(t)=T \psi(t)$ is an upper solution of problem (P1) and we get for all $q \in[p, 1)$

$$
\bar{\sigma}(t)=T \psi(t)=\frac{B t^{\beta+m-1}(\beta+m-t)}{\Gamma(\beta+m+1)} \geq \frac{B}{\Gamma(\beta+m)},
$$

and

$$
\begin{aligned}
& -{ }^{C} D_{1^{-}}^{q} \psi(t)+f(t, T \psi(t)) \\
= & \frac{-B}{\Gamma(2-q)}(1-t)^{1-q}+f(t, T v(t)) \\
\geq & -B(1-t)^{1-q}+f(t, T v(t)) \geq 0 .
\end{aligned}
$$

Moreover, we have $\psi(t) \leq \varphi(t)$. Then $\bar{\sigma}(t) \leq \underline{\sigma}(t)$, which implies that the upper and lower lower solutions $\bar{\sigma}$ and $\underline{\sigma}$ are in reverse order.

Step 2. Localization of solutions of problem (P1).

Define a sequence of modified problems $\left((P 4)_{q}\right), q \in[p, 1)$ by:

$$
\left((P 4)_{q}\right)\left\{\begin{array}{c}
{ }^{C} D_{1^{-}}^{q} v(t)=f((t, T \min [\varphi,(\max (v, \psi))])(t)), 0 \leq t \leq 1 \\
v(1)=0 .
\end{array}\right.
$$

Let us prove if $v_{q}$ is a solution of $\left((P 4)_{q}\right)$ for $q \in[p, 1)$, then

$$
\psi(t) \leq v_{q}(t) \leq \varphi(t), 0 \leq t \leq 1
$$

Set $w(t)=v_{q}(t)-\varphi(t)$. Then the initial condition implies $w(1)=0$. Assuming the contrary, we see that there exists $t_{1} \in\left[0,1\left[\right.\right.$ such that $w\left(t_{1}\right)>0$. By the continuity of $w$, we conclude the existence of two points $t_{2}$ and $t_{3}$ in the neighborhood of $t_{1}$ such that $w\left(t_{2}\right)=0$ and $w(t) \geq 0, t \in$ $\left[t_{3}, t_{2}\right]$. Now, it suffices to apply the right Caputo fractional derivative and take into account that 
$\varphi(t)=D_{0^{+}}^{\beta+m-1} \underline{\sigma}(t)$, properties (2.4)-(2.5) and the definition of lower solution (see Remark 2.3), to get

$$
\begin{aligned}
{ }^{C} D_{1^{-}}^{q} w(t) & ={ }^{C} D_{1^{-}}^{q} v_{q}(t)-{ }^{C} D_{1^{-}}^{q} \varphi(t) \\
& =f\left(t,\left(T \min \left[\varphi,\left(\max \left(v_{q}, \psi\right)\right)\right]\right)(t)\right)-{ }^{C} D_{1^{-}}^{q} \varphi(t) \leq 0
\end{aligned}
$$

for $t \in\left[t_{3}, t_{2}\right]$ and for all $q \in[p, 1)$. By Theorem 2.1, we deduce that $w$ is increasing on $\left[t_{3}, t_{2}\right]$. Since $w\left(t_{2}\right)=0$, we conclude that $w(t) \leq 0, t \in\left[t_{3}, t_{2}\right]$, which leads to a contradiction. Similarly, we obtain that $\psi(t) \leq v_{q}(t)$ on $t \in[0,1]$.

Now, from (2.8), we see that if $v=v_{p}$ is a solution of $\left((P 4)_{p}\right)$, then

$$
{ }^{C} D_{1^{-}}^{p} v(t)+f(t, T v(t))=0
$$

It follows that $u=T v$ is a solution of (P1). Finally, by applying operator $T$ to inequalities in (2.8), we find that

$$
\bar{\sigma}(t) \leq u(t) \leq \underline{\sigma}(t), 0 \leq t \leq 1
$$

and

$$
D_{0^{+}}^{\beta+m-1} \bar{\sigma}(t) \leq D_{0^{+}}^{\beta+m-1} u(t) \leq D_{0^{+}}^{\beta+m-1} \underline{\sigma}(t), 0 \leq t \leq 1
$$

Step 3. Existence of solutions of problem (P1).

Note that function $f$ is continuous. From Arzela-Ascoli theorem and the Schauder fixed point theorem, we show that the sequence of modified problems $\left((P 4)_{q}\right)$ has at least one solution for all $q \in[p, 1)$ and so $u=T v_{p}$ is a solution of (P1).

The proof of Theorem 2.4 is completed.

Now, we present two examples to illustrate our results.

Example 2.5. Consider problem (P1) with $\alpha=\frac{5}{3}, \beta=\frac{4}{3}$ and

$$
f(t, x)=x(1-t)^{\frac{1}{3}}, 0 \leq t \leq 1, x \in \mathbb{R} .
$$

Then $p=\alpha-m+1=\frac{2}{3}$. Choosing $A=0.1$ and $B=-0.1$, we see that Hypotheses (H1) and (H2) are satisfied. Indeed

$$
\begin{aligned}
f(t, x) & =x(1-t)^{\frac{1}{3}}=x(1-t)^{1-p} \leq A(1-t)^{1-q}, q \in\left[\frac{2}{3}, 1\right), \\
0 & \leq t \leq 1,0 \leq x \leq \frac{A}{\Gamma(\beta+m)}
\end{aligned}
$$


and

$$
\begin{aligned}
f(t, x) & =x(1-t)^{1-p} \geq \frac{B}{\Gamma(\beta+m)}(1-t)^{1-p} \\
& \geq B(1-t)^{1-q}, q \in\left[\frac{2}{3}, 1\right), \\
0 & \leq t \leq 1, \frac{B}{\Gamma(\beta+m)} \leq x \leq 0 .
\end{aligned}
$$

The expressions of lower and upper solutions are respectively

$$
\begin{aligned}
& \underline{\sigma}(t)=\frac{A t^{\beta+m-1}(\beta+m-t)}{\Gamma(\beta+m+1)}=\frac{t^{\frac{7}{3}}\left(\left(\frac{10}{3}\right)-t\right)}{10 \Gamma\left(\frac{7}{3}\right)}, \\
& \bar{\sigma}(t)=\frac{B t^{\beta+m-1}(\beta+m-t)}{\Gamma(\beta+m+1)}=\frac{-t^{\frac{7}{3}}\left(\left(\frac{10}{3}\right)-t\right)}{10 \Gamma\left(\frac{7}{3}\right)}
\end{aligned}
$$

Example 2.6. Take function $f$ in Example 2.5 as

$$
f(t, x)=\frac{-(1-t)^{\frac{1}{3}}}{10}, 0 \leq t \leq 1, x \in \mathbb{R} .
$$

Then (H1) and (H2) are satisfied. Moreover we have the expression of the solution

$$
u(t)=\frac{\Gamma\left(\frac{4}{3}\right) t^{\frac{7}{3}}\left(1-\frac{3}{10} t\right)}{10 \Gamma\left(\frac{10}{3}\right)}
$$

\section{REFERENCES}

[1] B. Ahmad, J. J. Nieto, Boundary value problems for a class of sequential integrodifferential equations of fractional order, J. Funct. Spaces Appl. 2013 (2013), Article ID 149659.

[2] O. P. Agrawal, Fractional variational calculus and the transversality conditions, J. Phys. A 39 (2006), 1037510384.

[3] D. Baleanu, K. Diethelm, E. Scalas, J.J. Trujillo, Fractional calculus models and numerical methods. Series on complexity, Nonlinearity and Chaos, World Scientific, Boston 2012.

[4] T. Blaszczyk, A numerical solution of a fractional oscillator equation in a non-resisting medium with natural boundary conditions, Romanian Reports in Phys. 67 (2015), 350-358.

[5] A. Guezane-Lakoud, R. Khaldi Successive approximations to solve higher order fractional differential equations, J. Nonlinear Funct. Anal. 2016 (2016), Article ID 29.

[6] A. Guezane-Lakoud, R. Khaldi, D. F. M. Torres, On a fractional oscillator equation with natural boundary conditions, Prog. Frac. Diff. Appl. in press, https://arxiv.org/pdf/1701.08962. 
[7] R. Khaldi, A. Guezane-Lakoud, Upper and lower solutions method for higher order boundary value problems, Progr. Fract. Differ. Appl. 3 (2017), 53-57.

[8] A. A. Kilbas, H. M. Srivastava, J. J. Trujillo, Theory and applications of fractional differential equations, North-Holland Mathematics Studies, Elsevier Science, Amsterdam, The Netherlands, 2006.

[9] Sotiris K. Ntouyas, Boundary value problems for nonlinear fractional differential equations and inclusions with nonlocal and fractional integral boundary conditions, Opuscula Math. 33 (2013), 117-138.

[10] I. Podlubny, Fractional Differential Equation, Academic Press, Sain Diego, 1999.

[11] S. G. Samko, A. A. Kilbas, and O. I. Marichev, Fractional integrals and derivatives, Theory and Applications, Gordon and Breach, Yverdon, Switzerland, 1993.

[12] C. De Coster, P. Habets, Two-point boundary value problems lower and upper solutions, Mathematics in Science and Engineering, Vol 205, Series Editor, C.K. Chui, Elsevier, 2006.

[13] D. Franco, Juan J. Nieto, D. O'Regan, Upper and lower solutions for first order problems with nonlinear boundary conditions, Extracta Mathematicae, 18 (2003), 153-160. 